\title{
Sex and longevity: Why women live longer than men
}

\author{
Milagros S. Peck ${ }^{\mathrm{a}}$, Amber T Porter ${ }^{\mathrm{a}}$, Breanna D Hayes ${ }^{\mathrm{a}}$, Dammah Otieno ${ }^{\mathrm{a}}$, Angelina J Hargrove ${ }^{\mathrm{a}}$, \\ Cynethia C Mayhand ${ }^{\mathrm{a}}$, Anisa I Johnson ${ }^{\mathrm{a}}$, Lynda M Brown ${ }^{\mathrm{a}}$
}

Sex differences in lifespan exist in most countries in the world, with women outliving men. This difference has existed for as long as records have been kept. This gap is not a uniquely human phenomenon; it exists in many animals as well. This is an important health issue that is not fully understood. Aging is a complex process. In this review we will examine the scientific basis for sex differences by focusing on four hypotheses that begin with examining why women are different from men. Men and women differ in our DNA. Women have two $\mathrm{X}$ chromosomes and men have an $\mathrm{X}$ and $\mathrm{Y}$ sex chromosome. Women have a second copy of the genes on the $\mathrm{X}$ chromosome which could be protective. Other hypotheses focus on the physiological difference between the sexes that result from the ratio of our sex hormones. Estrogen physiology changes women's body shape, affects the major organs like the heart and as a result impacts longevity. Sex differences in longevity are important because this becomes a lens for us to examine nutrition, health and wellness.

Keywords: sex differences; longevity; estrogen; testosterone

\section{Introduction}

Sex differences in lifespan exist in most countries in the world, with women outliving men by more than a decade in some countries (Neal-Perry, Nejat, \& Dicken, 2010; Regan \& Partridge, 2013). This difference has existed for as long as records have been kept (University of California). This gap is not a uniquely human trend; most animal have sex differences in aging (Austad, 2006). Despite documentation that women and most female animals love longer than males, we do not fully understand this phenomenon.

Aging is a process that affects us at the cellular, organism and species level. It is a principle factor in all major causes of death and an important part of examining health. In this review we will examine some of the cellular and physiological basis for sex differences in aging. Not all women outlive men; there are individual differences in lifestyle and health that impact us. Those differences are beyond the scope of this review. In this review we will primarily focus on the biology of aging and the physiological differences that exist between men and women. We will also discuss a theory based on the interplay between reproduction and longevity, the Grandmother Hypothesis. The Grandmother Hypothesis proposes that the wisdom of older women helps subsequent generations and can increase the health and wellbeing of upcoming generations.

\section{Why do women live longer than Men?}

At the cellular level the DNA of men and women differ and this is the basis of the first longevity hypothesis. Women have two $\mathrm{X}$ sex chromosomes and men have an $\mathrm{X}$ and a $\mathrm{Y}$ sex chromosome. The second $\mathrm{X}$ chromosome in women is protective when recessive mutations occur. Men will express recessive mutations on the $\mathrm{X}$ chromosome, and women have another $\mathrm{X}$ chromosome which gives us another copy of the genes on the $\mathrm{X}$ chromosome. If there is one mutated recessive gene and one normal gene in women, we will not express the mutation and are saved from the mutation. Women have an advantage because the phenotype or expression of an X-linked mutation would be prevented. This is the basis for the Heterogametic Hypothesis (Austad, 2006).

Hypothesis 1: The Heterogametic Hypothesis proposes a protective effect of the second X chromosome in women which protects them from the phenotype of X-linked recessive genes.

\section{Physiology of Estrogen and Testosterone}

The next two hypotheses focus on the physiology of the estrogen and testosterone. Sex hormones are what cause sexual dimorphic body changes starting in puberty. Additionally, these hormones have metabolic and cellular affects that could change how we age. Estrogen has positive effects on the inflammatory response, lipid profiles, the immune system and cardiovascular disease (CVD) (Hill et al., 2001; Palmer \& Clegg, 2015). To understand the physiology of the gonadal hormones, estrogen and testosterone, we begin with some of the biochemical foundations of the hormones.

Cholesterol is the precursor for testosterone and estrogens. Structurally hormones derived from cholesterol that retain the four-ring backbone are steroid hormones. It is not surprising then that these hormones would then affect cholesterol synthesis in ways that impact heart disease risk in men and women. Hormones often form feedback loop pathways with their precursor compounds. Estrogen reduces low density lipoproteins (LDL) and increases high density lipoprotein (HDL) cholesterol creating a positive lipid profile in women (Hill et al., 2001). This is one of the mechanisms for the proposed benefit of high estrogen levels in women. Unfortunately, testosterone or low estrogen levels have the opposite effect on cholesterol levels which then increases heart disease risk in men and postmenopausal women (Carr, 2003).

a. Department of Family and Consumer Sciences, Food and Nutritional Sciences Program, North Carolina Agricultural \& Technical State University, Greensboro, NC, 27401 
The final step in estrogen synthesis is the conversion of testosterone to estradiol, the main estrogen in our bodies (Figure 1). Men and women have testosterone and estrogen, the difference is the amounts of each hormone. Young women have higher estrogen levels then men or postmenopausal women. Inflammation is a factor that increases the risk of the metabolic syndrome which includes obesity, insulin resistance, diabetes, unhealthy blood lipid levels and hypertension (Carr, 2003; Lee et al., 2009). The metabolic syndrome is a risk factor for cardiovascular disease.

Figure 1: Testosterone converts to Estradiol

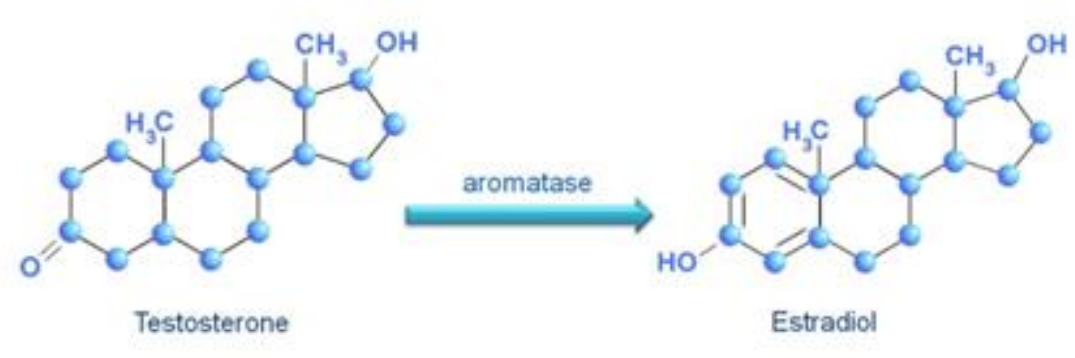

Figure 1 In the final reaction Estrogen synthesis testeosterone is converted by the enzyme aromatase to Esterdiol, the principal estrogen in our bodies. Men and women have both hormones just different levels of each.

Estrogen strengthens the immune system and decreases oxidative stress (Austad, 2006). Oxidative stress results when free radicals cause damage to the body. A free radical is an atom with an unpaired electron that acts as a weapon in the body. Healthy atoms have paired electrons. Oxygen, ultraviolet light, iron and other pro-oxidants in our environment have the ability to steal an electron from healthy atoms which weaponizes them as free radicals. Our immune system can use free radicals to kill viruses and bacteria, or the free radical can harm healthy cells in its pursuit of an electron to make itself whole. Antioxidants like vitamins A, C and E or minerals selenium, copper and zinc have the ability to donate an electron to quench free radicals without weaponizing.

Hypothesis 2: Estrogen strengthens the immune system and decreases oxidative stress.

Antioxidants exist in pathways that donate electrons to prevent them from becoming free radicals. We cannot prevent free radicals from developing in our bodies. They are produced in our energy pathways as glucose is transformed into ATP energy when we eat. We are also surrounded by oxygen and must breath to live. To be healthy we must balance free radicals with enough antioxidants to avoid imbalance. Antioxidant vitamins and minerals are abundant in fresh fruit and vegetables which is why the USDA My Plate healthy eating guide recommends that half your plate be

Figure 2: Pear and Apple Body Shapes

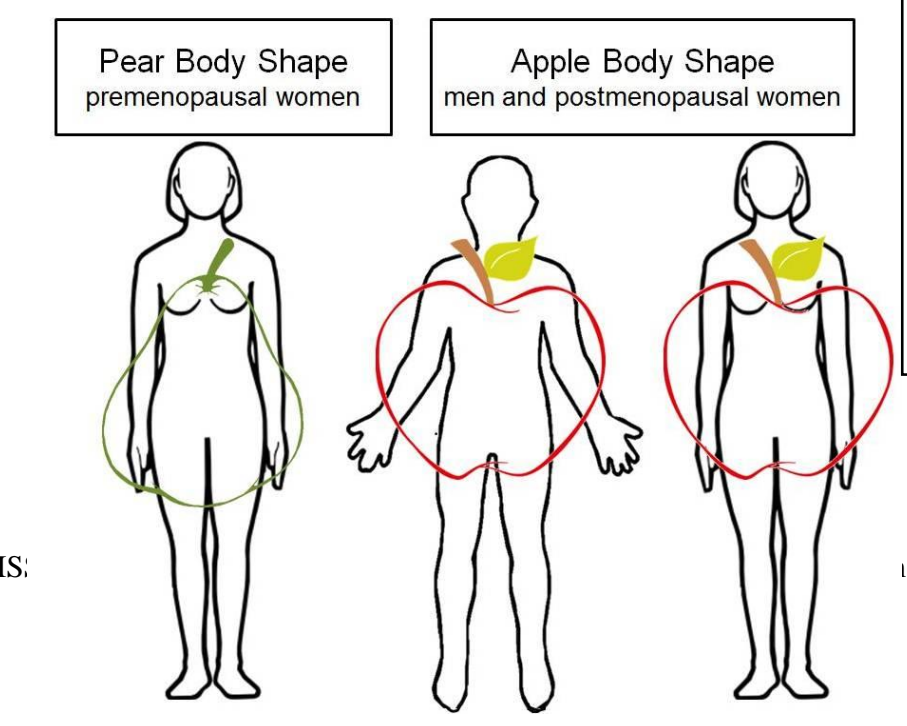

fruits and vegetables (Mathers, 2013). In a study that examined the antioxidant activity in the mitochondria of women and men, women had higher antioxidant activity (Borras et al., 2003). Lower oxidative stress in women would translate into healthier immune systems. This could be a reason that women have lower risk of chronic diseases than men. In reviewing the top 12 causes of death in the US, men have a higher incidence of each of the major causes of death than women (Chang, Moonesinghe, Athar, \& Truman, 2015). That includes cardiovascular disease and goes beyond it to accidents, cancer and diabetes.

Estrogen determines fat depot distribution (Palmer \& Clegg, 2015). The accumulation of visceral fat is a risk factor for cardiovascular disease (CVD) (Carr, 2003) which is the number one cause of death in men and women in the US (Chang et al., 2015). Estrogen promotes the accumulation of subcutaneous fat in young women which is called the pear fat distribution (Figure 2) (Palmer \& Clegg, 2015). When men gain weight, it is more likely that the excess fat occurs in the abdominal area or the apple distribution [see Figure 1] (Palmer \& Clegg, 2015). Abdominal fat releases hormones that increase inflammation, and inflammation increases the risk of CVD (Brown \& Clegg, 2010; Carr, 2003).

Postmenopausal women have lower levels of estrogen and this is related to an increase in visceral fat (Carr, 2003; Lee et al., 2009; Palmer \& Clegg, 2015) changing their fat distribution to the apple shape (Figure 2). As a result, postmenopausal women have the same CVD risk as agematched men (Brown \& Clegg, 2010; Carr, 2003).
Figure 2: Young women most often accumulate fat below the waistline in the hips and buttocks. This is known as the pear fat distribution. In people with lower estrogen levels, like men and postmenopausal women fat accumulates above the waist. This is known as the apple fat distribution. The apple fat distribution is associated with a higher risk of heart disease. After menopause the risk of heart disease in women matches that of age-matched men. 
Estrogen has many beneficial physiological effects and in some cases testosterone has opposing effects. How is this possible when they are only one reaction apart? In medicinal chemistry the difference between the two molecules is great. Structure determines function. Another way to approach this question is to ask; would men live longer or be healthier with lower testosterone levels? There are instances recorded from the Korean Royal Court from the $16^{\text {th }}$ to the $18^{\text {th }}$ century that eunuchs were stronger and healthier than intact Korean men and lived 14-19 years longer (Min, Lee, \& Park, 2012). Longer lives have also been recorded in experimental and farm animals following castration. How would loss of reproductive capacity extend life? There is a theory that reproduction and longevity are energy requiring processes that can interact. In the Disputable Soma hypothesis it is proposed that when reproduction is stopped there would then be more energy to strengthen the immune system (Westendorp \& Kirkwood, 1998).

Another version of the reproduction and longevity interplay is that women live longer than men because men remain fertile all their lives and women go through menopause. The average age for menopause is 51 years old and the life span of US women is 78 years old. That means many women may live 27 years beyond their reproductive years. There are other animals that live beyond their reproductive years like whales and elephants. In these animals older females are a repository for wisdom that is protective the upcoming generations and the Grandmother Hypothesis (Figure 3) proposes that this is true for humans (Hawkes \& Coxworth, 2013; Wainer-Katsir, Zou, \& Linial, 2015).

When Dave Buettner examined the Blue Zones or areas of the world where people routinely live past 100 years and remain healthy through their lives (Buettner). Buettner noted that children are healthier when the grandparents are part of their lives. This also gives credence to the Grandmother Hypothesis (Figure 3). In the pictorial representation we see a pregnant woman at the top of the figure who must work and balance a family once that child is born. At the bottom we see that if a Grandmother is part of the family it can help to ease the work load and it is possible that the mother would another child sooner and the process continues. This is beneficial for the mother because she may have children at a younger age. Medically any woman who has children past the age of 35 is in a high risk pregnancy.

Hypothesis 4: The Grandmother Hypothesis proposes that the wisdom of older women is protective for the upcoming generations.

Figure 3: The Grandmother Hypothesis

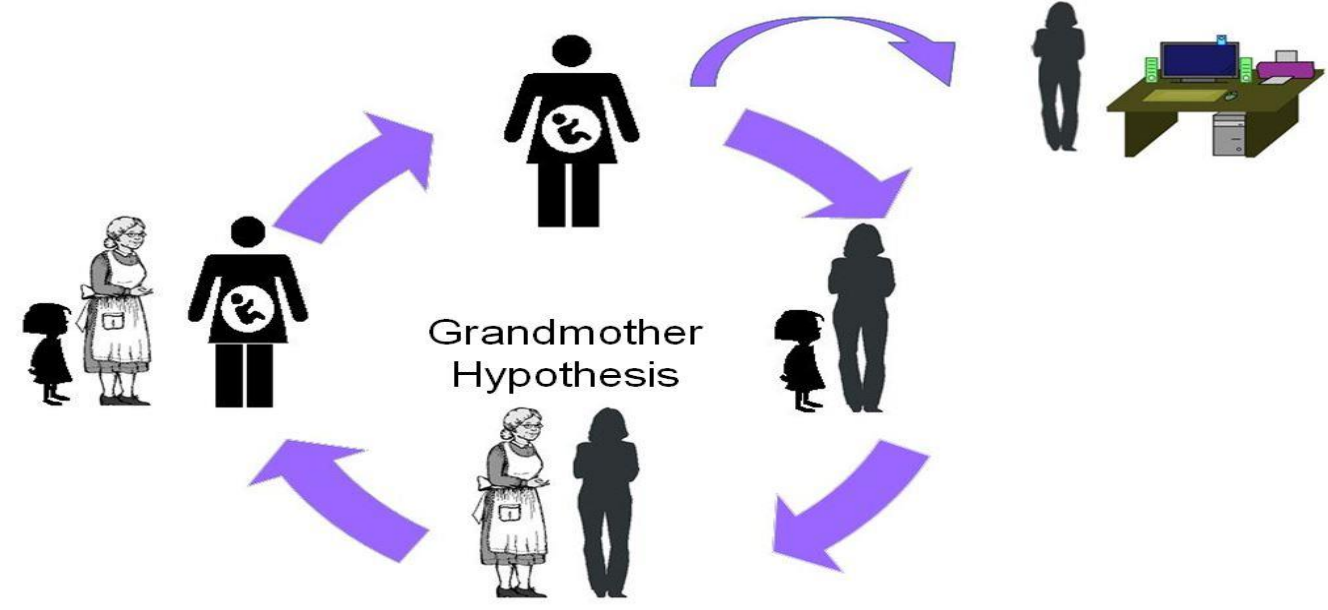

Figure 3: At the top we see that once a woman has children she may have to balance her work and home life. The Grandmother hypothesis proposes that the wisdom of older women is helpful to children and grandchildren. An example would be at the bottom we see the presence of the Grandmother may help the mother have more children earlier. There is evidence that grandchildren are healthier when they have grandparents in their lives. 


\section{Summary}

Figure 4: Hypotheses for the Longevity of Women

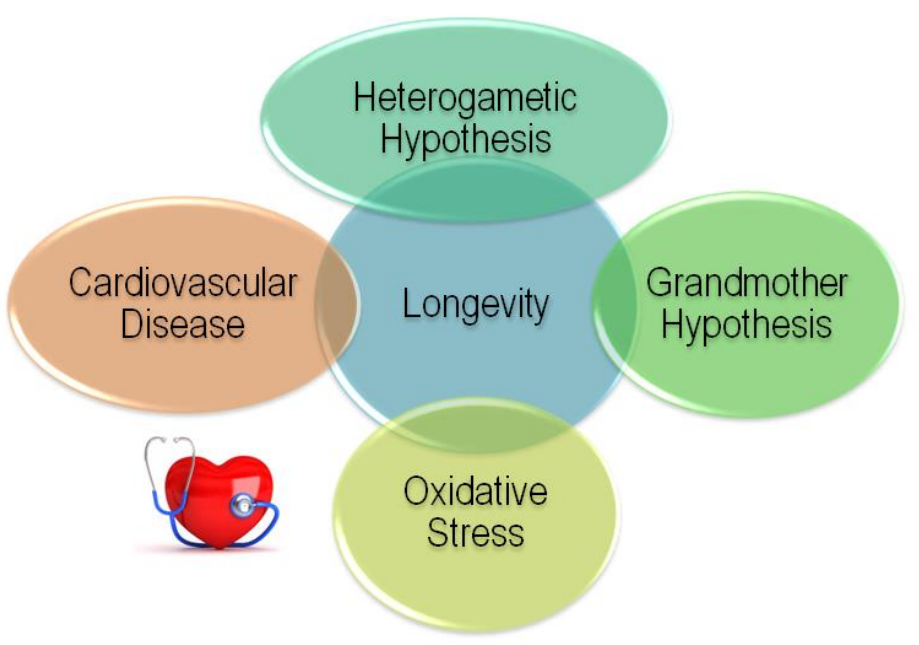

Figure 4: No single hypothesis for the sexual dimorphism in lifespan fully explains why women live longer than men. However if there is a small advantage from the hypotheses presented in this review it is possible to project that as a group women would live longer than men.

We have known that women as a group live longer than men yet the reasons for this have not been fully explained. This phenomenon exists throughout the world and across most animal species demonstrating that this is not an anomaly but a robust scientific paradox. In this review we explored the literature to get a better understanding for the longevity of women. The four hypotheses explored here explain some portion of that difference, but no one hypothesis is sufficient or universally accepted (see Figure 4).

We found connections between the biological reasons that women are women, like the $\mathrm{XX}$ chromosomes and predominance of estrogen in our bodies, and longevity. While men have estrogen and women have testosterone, it is the predominant sex hormone that seems to determine health. This is somewhat surprising when we look at the similarity of estrogen and testosterone. These hormones share a cholesterol backbone and are one reaction apart.

Finally we reviewed the interplay between reproduction and longevity in the Grandmother Hypothesis. It is possible that women live longer because our reproductive capacity ends and almost $1 / 3$ of our lives this energy is available to strengthen our immune systems.

As with all good research questions, in reading through the literature we discovered there are gaps in our knowledge of sex differences, the physiology of sex hormones and longevity. This is good news because research involves identifying gaps in our current knowledge in order to develop hypotheses to increase our knowledge. Our quest was to understand sex differences in longevity and our hope is that one day this will be understood in ways that will increase the health of all people.

\section{Acknowledgements}

We gratefully acknowledge the FCS 640 Geriatric Nutrition class for their contributions that started this manuscript: Alani Adkins, Samirah Alotaibi, Aria Cunningham, Shu Fang, Rahmos Long, Sherri Ray, Joanna Siaw, Demetrius Smalls, Michael Weaver, Johnesha Williams and Keshanna Williams. This work was supported in part by a North Carolina Agricultural \& Technical State University USDA Evans Allen Grant (LMB).

\section{Disclosures}

The authors have no conflicts of interest to declare.

\section{References}

Austad, S. N. (2006). Why women live longer than men: sex . Gend Med, 3(2), 79-92.

Borras, C., Sastre, J., Garcia-Sala, D., Lloret, A., Pallardo, F. V., \& Vina, J. (2003). Mitochondria from females exhibit higher antioxidant gene expression and lower oxidative damage than males. Free Radic Biol Med, 34(5), 546-552.

Brown, L. M., \& Clegg, D. J. (2010). Central effects of estradiol in the regulation of food intake, body ity. J Steroid Biochem Mol Biol, 122(1-3), 65-73. doi: 10.1016/j.jsbmb.2009.12.005

Buettner, Dan. (2012). The Blue Zones : 9 lessons for living longer from the people who've lived the longest (Second edition. ed.).

Carr, M. C. (2003). The emergence of the metabolic syndrome with menopause. $J$ Clin Endocrinol Metab, 88(6), 2404-2411.

Chang, M. H., Moonesinghe, R., Athar, H. M., \& Truman, B. I. (2015). Trends in Disparity by Sex and Race/Ethnicity for the Leading Causes of Death in 
the United States-1999-2010. J Public Health Manag Pract. doi: 10.1097/PHH.0000000000000267

Hawkes, K., \& Coxworth, J. E. (2013). Grandmothers and the evolution of human longevity: a review of findings and future directions. Evol Anthropol, 22(6), 294302. doi: 10.1002/evan.21382

Hill, K., Boesch, C., Goodall, J., Pusey, A., Williams, J., \& Wrangham, R. (2001). Mortality rates among wild chimpanzees. J Hum Evol, 40(5), 437-450. doi: 10.1006/jhev.2001.0469

Lee, C. G., Carr, M. C., Murdoch, S. J., Mitchell, E., Woods, N. F., Wener, M. H., . . . Brunzell, J. D. (2009). Adipokines, inflammation, and visceral adiposity across the menopausal transition: a prospective study. J Clin Endocrinol Metab, 94(4), 1104-1110.

Mathers, J. C. (2013). Nutrition and ageing: knowledge, gaps and research priorities. Proc Nutr Soc, 72(2), 246250. doi: 10.1017/S0029665112003023

Min, K. J., Lee, C. K., \& Park, H. N. (2012). The lifespan of Korean eunuchs. Curr Biol, 22(18), R792-793. doi: 10.1016/j.cub.2012.06.036
Neal-Perry, G., Nejat, E., \& Dicken, C. (2010). The neuroendocrine physiology of female reproductive aging: An update. Maturitas, 67(1), 34-38. doi: 10.1016/j.maturitas.2010.04.016

Palmer, B. F., \& Clegg, D. J. (2015). The sexual dimorphism of obesity. Mol Cell Endocrinol, 402, 113-119. doi: 10.1016/j.mce.2014.11.029

Regan, J. C., \& Partridge, L. (2013). Gender and longevity: why do men die earlier than women? Comparative and experimental evidence. Best Pract Res Clin Endocrinol Metab, 27(4), 467-479. doi: 10.1016/j.beem.2013.05.016

University of California, Berkeley.). The Human Mortality Database. from http://www.mortality.org

Wainer-Katsir, K., Zou, J. Y., \& Linial, M. (2015). Extended fertility and longevity: the genetic and epigenetic link. Fertil Steril, 103(5), 1117-1124. doi: 10.1016/j.fertnstert.2015.02.008

Westendorp, R. G., \& Kirkwood, T. B. (1998). Human longevity at the cost of reproductive success. Nature, 396(6713), 743-746. doi: 10.1038/25519 